\title{
Synthesis of Hydrophobic Mesoporous Material MFS and Its Adsorption Properties of Water Vapor
}

\author{
Guotao Zhao, ${ }^{1}$ Zhenxiao Zhao, ${ }^{2}$ Junliang Wu, ${ }^{1}$ and Daiqi Ye ${ }^{1}$ \\ ${ }^{1}$ College of Environment and Energy, South China University of Technology, Guangzhou 510006, China \\ ${ }^{2}$ School of Chemistry and Chemical Engineering, Guangxi University, Nanning 530004, China \\ Correspondence should be addressed to Daiqi Ye; cedqye@scut.edu.cn
}

Received 25 April 2014; Accepted 18 May 2014; Published 3 June 2014

Academic Editor: Qingrui Zhang

Copyright (c) 2014 Guotao Zhao et al. This is an open access article distributed under the Creative Commons Attribution License, which permits unrestricted use, distribution, and reproduction in any medium, provided the original work is properly cited.

\begin{abstract}
Fluorine-containing hydrophobic mesoporous material (MFS) with high surface area is successfully synthesized with hydrothermal synthesis method by using a perfluorinated surfactant SURFLON S-386 template. The adsorption properties of water vapor on the synthesized MFS are also investigated by using gravimetric method. Results show that SEM image of the MFS depicted roundish morphology with the average crystal size of $1-2 \mu \mathrm{m}$. The BET surface area and total pore volume of the MFS are $865.4 \mathrm{~m}^{2} \mathrm{~g}^{-1}$ and $0.74 \mathrm{~cm}^{3} \mathrm{~g}^{-1}$ with a narrow pore size distribution at $4.9 \mathrm{~nm}$. The amount of water vapor on the MFS is about $0.41 \mathrm{mmol} \mathrm{g}^{-1}$ at $303 \mathrm{~K}$, which is only $52.6 \%$ and $55.4 \%$ of MCM- 41 and SBA-15 under the similar conditions, separately. The isosteric adsorption heat of water on the MFS is gradually about $27.0-19.8 \mathrm{~kJ} \mathrm{~mol}^{-1}$, which decreases as the absorbed water vapor amount increases. The value is much smaller than that on MCM-41 and SBA-15. Therefore, the MFS shows more hydrophobic surface properties than the MCM-41 and SBA-15. It may be a kind of good candidate for adsorption of large molecule and catalyst carrier with high moisture resistance.
\end{abstract}

\section{Introduction}

With the development of industry, air pollution and its adverse impact on the environment and human health has been an ongoing issue for decades, especially in developing countries. The growing world population and the emergence of recently industrialized countries make this issue even more pronounced. Volatile organic compounds (VOCs) are the main pollutants in the ambient air released by chemical, petrochemical, and related industries and have drawn more and more attention in recent years. Release of VOCs into the atmosphere may significantly reduce air quality and do harm to our health and welfare directly [1]. In addition, VOCs are key reactants involved in photochemical reactions occurring in the atmosphere, which lead to serious environmental hazards when they reach a certain concentration [2]. So, the VOCs concentration in air needs to be strictly regulated to an extremely low level and an efficient method to reduce the concentration in atmosphere is now strongly demanded.

There are many techniques available to abate the emission of VOCs, such as adsorption [3], catalytic oxidation [4], condensation [5], membrane separation [6], and biological treatments. Catalysis method has been considered as one of the most cost-effective and environmentally friendly technologies for the removal of VOCs, especially at high concentrations.

Mesoporous materials, with a pore size range of $2-50 \mathrm{~nm}$, offer unique opportunities to catalysis thanks to the large surface area. The wide range of acid/base or redox catalysts will be developed after loading some active elements inside the pore walls of such materials [7, 8]. Mesoporous inorganic materials possess high specific surface area, nanometersized channels [9] with an ordered interconnected internal structure, and high thermal stability [10], which has made feasible potential applications as the carrier of many kinds of catalysts [11]. Recently, the ordered mesoporous silica MCM41 [12], MCM-48 [13], SBA-15 [14], SBA-16 [15], and SBA-12 [16] have been investigated to introduce active elements to prepare the catalysts.

However, water vapor is often present in polluted air under the real situations and may affect the catalytic characteristics of catalysts. Sometimes, water will become a poison 
for the catalytic combustion of VOCs [17]. Zelenak et al. [15] investigated the effect of water vapor on the activity of copper loaded catalysts with different supports such as $\mathrm{CuO} / \gamma$ $\mathrm{Al}_{2} \mathrm{O}_{3}, \mathrm{CuO} / \mathrm{SiO}_{2}$, and $\mathrm{CuO} / \mathrm{TiO}_{2}$ for styrene oxidation and found that the presence of water vapor had a significant negative effect on the activity of the copper based catalysts for styrene oxidation. Li et al. [18] reported the catalytic activity of copper and manganese based catalysts prepared with different supports for toluene oxidation. Results showed that the conversion of toluene decreased by about $90 \%$ on the $\mathrm{CuMn}(1) \mathrm{O}_{x} / \gamma-\mathrm{Al}_{2} \mathrm{O}_{3}$ and $50 \%$ on the $\mathrm{CuMn}(1) \mathrm{O}_{x} / \mathrm{TiO}_{2}$ in the presence of water vapor $(3.78, \mathrm{vol} \%)$ at $180^{\circ} \mathrm{C}$. Jacobs et al. [19] studied the effects of water on the deactivation of Pt-promoted $\mathrm{Co} / \mathrm{Al}_{2} \mathrm{O}_{3}$ catalyst by XAFS and found that the catalyst activity depicted a sudden irreversible loss when the humidity reached $25 \%$. Therefore, the improvement of the water resistance of catalyst supports by different methods of surface treatment is the key issue in practice applications. Xia et al. [20] prepared a Pt supported on MCM-41 catalyst synthesized in the presence of fluoride anions for combustion of toluene and discovered that the presence of the additional $\mathrm{H}_{2} \mathrm{O}$ had almost no effect on the oxidation activity of this catalyst. The synthesized MCM-41 support showed the more hydrophobic properties than the common MCM-41 without adding fluoride anions. Thus, it can be seen that adding fluoride element may be a promising way to strengthen the hydrophobicity of materials.

In this work we proposed to use a novel template (perfluorinated-surfactant SURFLON S-386, polymeric perfluorocarboxylic acid) to synthesize a more hydrophobic mesoporous MFS with higher surface area. The element of fluorine was successfully introduced into the structure of the mesoporous MFS and formed a more hydrophobic surface on it. The adsorption performance of water vapor at different temperatures on the MFS was investigated and compared with traditional mesoporous materials MCM-41 and SBA15. Meanwhile, the water isosteric heat of adsorption on the synthesized MFS was estimated and the interaction between water molecules and the MFS was studied.

\section{Experimental}

2.1. Synthesis of the MFS Material. The MFS samples were synthesized using SURFLON S-386 ( $\geq 99 \%$, ASAHI glass) and tetraethylorthosilicate ( $>98 \%$, J\&K Scientific, TEOS) under acidic conditions from a hydrothermal reaction. First, SURFLON S-386 $(0.4,1.0$, and $1.6 \mathrm{~g})$ was dissolved into $120 \mathrm{~mL}$ deionized water at $313 \mathrm{~K}$ in a $250 \mathrm{~mL}$ beaker. After strong stirring for $60 \mathrm{~min}$, TEOS $(10.0 \mathrm{~g})$ and aqueous $\mathrm{HCl}$ ( $5 \mathrm{~mL}, 1 \mathrm{~mol} \mathrm{~L}^{-1}$, Donghong) were added to the solution and stirred for $30 \mathrm{~min}$. The derived homogenous solution was then transferred into an autoclave and aged for $24 \mathrm{~h}$ in air to produce a rigid gel. The gel was covered and heated at $373 \mathrm{~K}$ for $48 \mathrm{~h}$. The obtained samples were centrifuged and then washed with water and alcohol. After that these samples were dried in an oven at $373 \mathrm{~K}$ for $12 \mathrm{~h}$. Finally, the samples were calcined at $823 \mathrm{~K}$ for $6 \mathrm{~h}$ with a heating rate of $1 \mathrm{~K} \mathrm{~min}^{-1}$. The obtained samples were named as MFS-01 (0.4 g SURFLON
S-386), MFS-02 (1.0 g SURFLON S-386), and MFS-03 (1.6 g SURFLON S-386), separately.

2.2. Materials Characterization. Scanning electron microscope (SEM) images were obtained on a Philips FEIXL30 operated by using a $10 \mathrm{kV}$ accelerating voltage after gold deposition. Transmission electron microscope (TEM) images were taken with a JEM-2010HR electron microscope operating at $200 \mathrm{kV}$. In TEM measurements, the samples were prepared by dispersing the powdered product as slurry in ethanol, after which they were dispersed and dried on a holey carbon film on a cooper grid. Small-angel X-ray diffraction (XRD) pattern was obtained with a D-MAX 2200 VPC using $\mathrm{CuK} \alpha$ radiation. Langmuir and Brunauer-EmmettTeller (BET) surface areas and pore volume of the synthesis MFS-02 were measured with a Micromeritics ASAP 2020 sorptometer using $\mathrm{N}_{2}$ adsorption at $77 \mathrm{~K}$. Fourier Transforminfrared spectroscopy (FT-IR) measurements were recorded on a Nicolet 6700 spectrophotometer.

\subsection{Measurements of Adsorption Isotherms of Water Vapor.} Water vapor adsorption on the synthesized MFS-02, MCM41 (from Novel chemical Technology Co.), and SBA-15 (from Novel chemical Technology Co.) at 283-313 K was performed gravimetrically in a water sorption analyzer (Aquadyne DVS, USA). Prior to each sorption experiment, approximately 20$30 \mathrm{mg}$ of preactivated sample (heated at $408-413 \mathrm{~K}$ under vacuum overnight) was charged into the sample pan with an accuracy of $\pm 0.1 \mu \mathrm{g}$ to acquire data. Microbalance system can provide a direct measurement of adsorption. The temperature and humidity of the sorption chamber can be controlled by thermal couple and a dehumidifier and a humidifier with an accuracy of $\pm 0.1 \mathrm{~K}$ and $\pm 0.1 \mathrm{RH} \%$, respectively.

2.4. Measurements of the Isosteric Heat of Adsorption. The isosteric heat of adsorption can be used to evaluate the interaction between the adsorbate molecules and the adsorbent surfaces. The isosteric heats of adsorption as a function of the surface coverage can be calculated from isotherms at different temperatures by using Clausius-Clapeyron equation (1) $[21,22]$ :

$$
\ln (P)=-\frac{\Delta H}{R T}+C
$$

where $\Delta H_{s}$ is isosteric heat of water vapor adsorption, $\mathrm{kJ}$ $\mathrm{mol}^{-1}, R$ is the ideal gas constant, $\mathrm{kJ} \mathrm{mol}{ }^{-1} \mathrm{~K}, P$ is the water vapor feed pressure, $\mathrm{Pa}$, and $C$ is an integration constant.

The isotherms of water vapor on the MFS-02 at different temperatures can be used to estimate the heat of adsorption [23]. Firstly, the water vapor isotherms were converted to water vapor adsorption isosteres. After that, $\ln P$ is plotted to $1 / \mathrm{T}$, and thus it yields a straight line with a slope $-\Delta H_{s} / R$. Finally, the isosteric heat $\left(\Delta H_{s}\right)$ of water vapor adsorption can be calculated directly from the slope $-\Delta H_{s} / R$ of the plotted straight line. It is similar to calculating isosteric heat of water vapor adsorption for MCM-41 and SBA-15. 


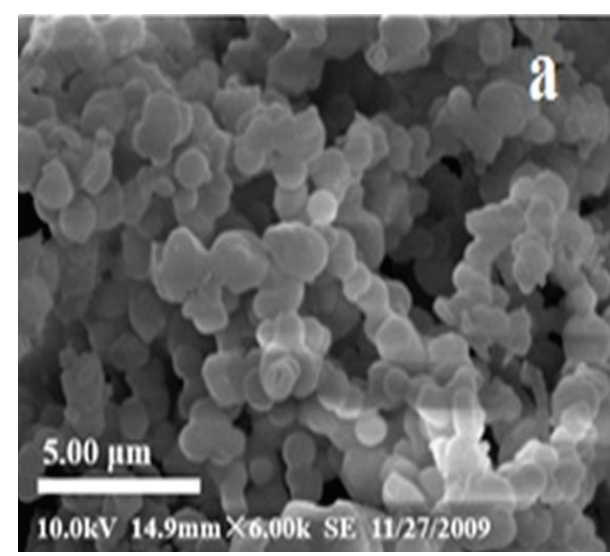

(a)

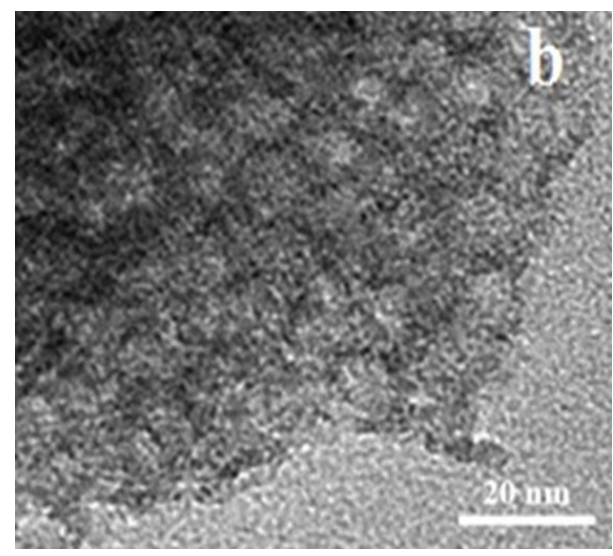

(b)

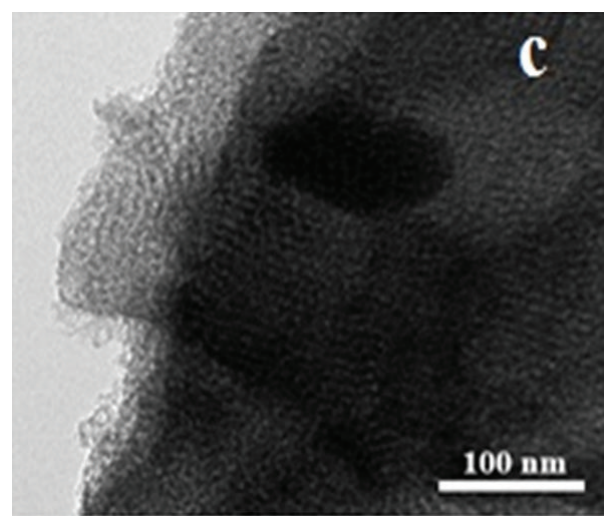

(c)

FIgURE 1: SEM (a) and TEM (b and c) images of the synthesis MFS-02.

\section{Results and Discussion}

\subsection{Samples Characterization}

(1) SEM and TEM. Figure 1(a) shows the SEM image of the MFS-02 sample. It can be seen that the MFS-02 shows a spherical-like morphological characteristic with a uniform diameter around 1-2 $\mu \mathrm{m}$.

Figures 1(b) and 1(c) show the TEM images of the MFS02 sample. Some clear amorphous channel displays on the surface of the material. The light and dark stripes of typical mesoporous structure can also be observed, revealing the presence of mesopores in the MFS [24].

(2) XRD. Figure 2 shows the small-angle X-ray diffraction (XRD) patterns of the MFS-01, MFS-02, and MFS-03 samples. It can be seen that only one diffraction peak was detected at $2 \theta=0.7^{\circ}$. The characteristic peak at low angle indicates a short-range order in the structure [24], which is consistent with the TEM images shown in Figures 1(b) and 1(c). Moreover, the intensity of the peak was very weak in XRD pattern of the MFS-01, indicating a weak crystallinity in the structure. With the increase of SURFLON S-386 content in the mother solution, the intensity of this peak was gradually increased firstly and then decreased. It was indicated that the appropriate amount of the template will favor to form a relative order structure and high crystallinity.

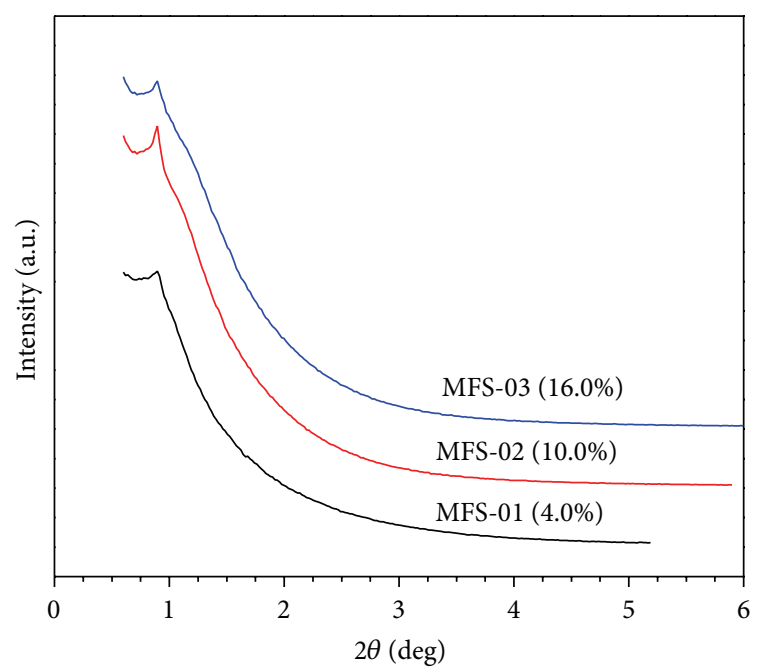

FIGURE 2: PXRD pattern of the synthesized MFS crystals.

(3) ASAP. The specific surface area was calculated according to the Brunauer-Emmett-Teller (BET) model and the average pore diameter was obtained according to the BarrettJoyner-Halenda (BJH) model. Figure 3(a) shows $\mathrm{N}_{2}$ adsorption/desorption isotherms of the MFS- 02 at $77 \mathrm{~K}$ and its pore size distribution of the MFS-02 crystals from BJH model. 


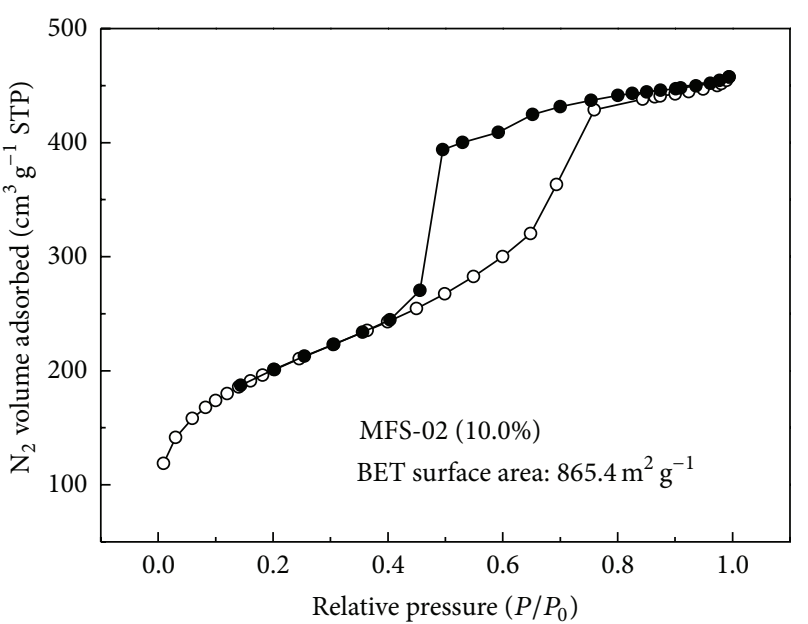

(a)

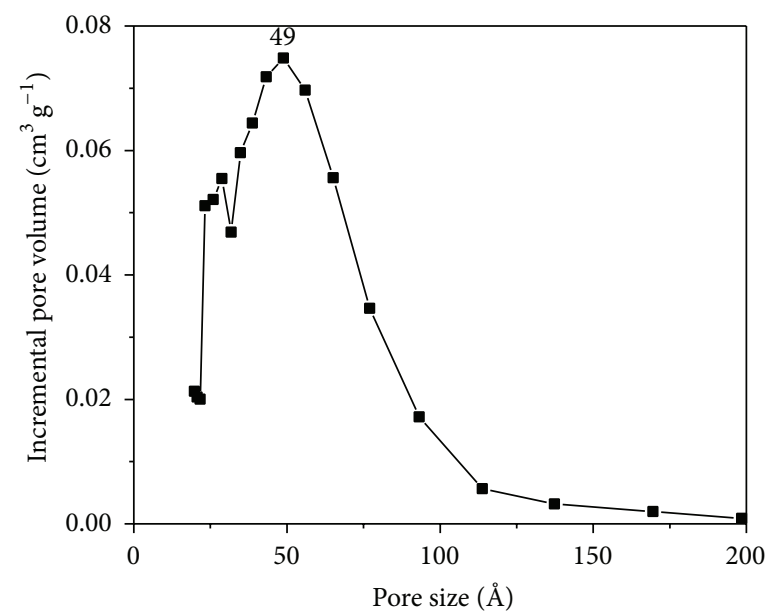

(b)

Figure 3: (a) $\mathrm{N}_{2}$ sorption isotherms of the MFS-02 crystals at $77 \mathrm{~K}$; (b) pore size distribution the MFS- 02 crystals from the BJH model. $P / P_{0}$ is the ratio of gas pressure $(P)$ to saturation pressure $\left(P_{0}=101.3 \mathrm{Kpa}\right)$.

TABLE 1: The parameters of the porous texture of the synthesized MFSs.

\begin{tabular}{lccc}
\hline $\begin{array}{l}\text { Sample } \\
\text { According to } \\
\begin{array}{l}\text { SURFLON S-386 } \\
\text { contents, \%) }\end{array}\end{array}$ & $\begin{array}{r}\text { BET Surface } \\
\text { Area }\left(\mathrm{m}^{2} \mathrm{~g}^{-1}\right)\end{array}$ & $\begin{array}{c}\text { Langmuir } \\
\text { Surface Area } \\
\left(\mathrm{m}^{2} \mathrm{~g}^{-1}\right)\end{array}$ & $\begin{array}{c}\text { Pore volume } \\
\left(\mathrm{cm}^{2} \mathrm{~g}^{-1}\right)\end{array}$ \\
\hline 4 & 563.9 & 785.7 & 0.74 \\
8 & 766.6 & 1062.7 & 0.64 \\
10 & 865.4 & 1205.3 & 0.75 \\
14 & 718.1 & 989.5 & 0.70 \\
24 & 544.2 & 760.2 & 0.78 \\
\hline
\end{tabular}

The $\mathrm{N}_{2}$ adsorption isotherm exhibits a reversible type IV isotherm with a clear hysteresis at $P>0.4 \mathrm{~atm}$, which is a characteristic of mesoporous material. Its BET surface area, Langmuir surface area, and pore volume were calculated to be $865.4-1205.1 \mathrm{~m}^{2} \mathrm{~g}^{-1}$ and $0.74 \mathrm{~cm}^{3} \mathrm{~g}^{-1}$, respectively. Its mesopore area and mesopore volume were $928.0 \mathrm{~m}^{2} \mathrm{~g}^{-1}$ and $0.7 \mathrm{~cm}^{3} \mathrm{~g}^{-1}$. In BJH differential pore volume plot, only one sharp peak is observed at about $4.9 \mathrm{~nm}$ for the MFS-02 crystals (Figure 3(b)), indicating a very narrow mesoporous material.

Table 1 shows the parameters of the porous texture of the series MFSs synthesized by using different amounts of the SURFLON S-386 template. It can be seen that the BET surface areas and Langmuir surface areas were increased gradually with the increase of the addition of SURFLON S-386 content. The surface areas reached the maximum when the SURFLON S-386 contents was up to $10 \%$ and then declined gradually. It indicates that concentration of template may create greater impact on the formation of micelles and crystalline phases. It is favourable for the formation of liquid crystalline phases as increase of the template under low concentration. However, agglomeration of surfactant molecules will appear at high

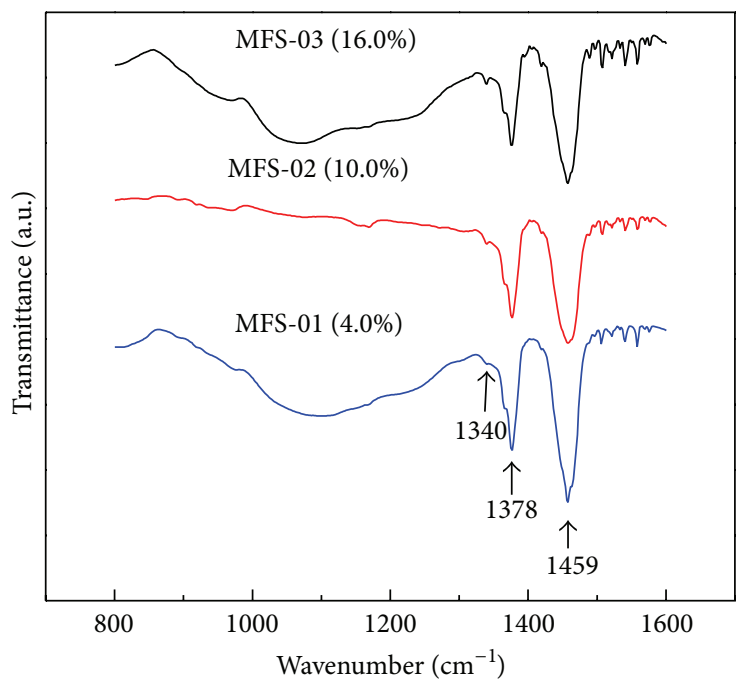

FIGURE 4: FT-IR spectra of MFSs with various dosage of SURFLON S-386 (a: MFS-01; b: MFS-02; c: MFS-03).

template concentration, which may affect the formation of liquid crystalline phases and mesoscopic structures selfassemble and then lead to the lower specific surface area of MFS ultimately.

(4) FTIR. Figure 4 shows the FTIR spectra of the MFS samples synthesized with different SURFLON S-386 contents. For each sample, the spectra show two strong distinct peaks at 1378 and $1459 \mathrm{~cm}^{-1}$ as well as and a weak peak at $1340 \mathrm{~cm}^{-1}$, respectively.

The peak at $1378 \mathrm{~cm}^{-1}$ is assigned to the symmetric deformation vibration of methyl groups $[25,26]$, which came from the residual of TEOS. The peak at $1459 \mathrm{~cm}^{-1}$ is associated with $-\mathrm{CF}_{2}$ asymmetric stretch vibration [27], and the peak at $1340 \mathrm{~cm}^{-1}$ represents $-\mathrm{CF}_{3}$ stretch vibration of 


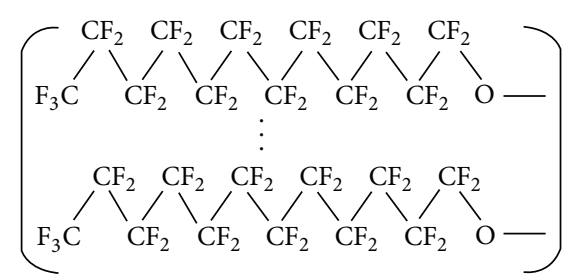

FIGURE 5: Molecular structure of SURFLON S-386.

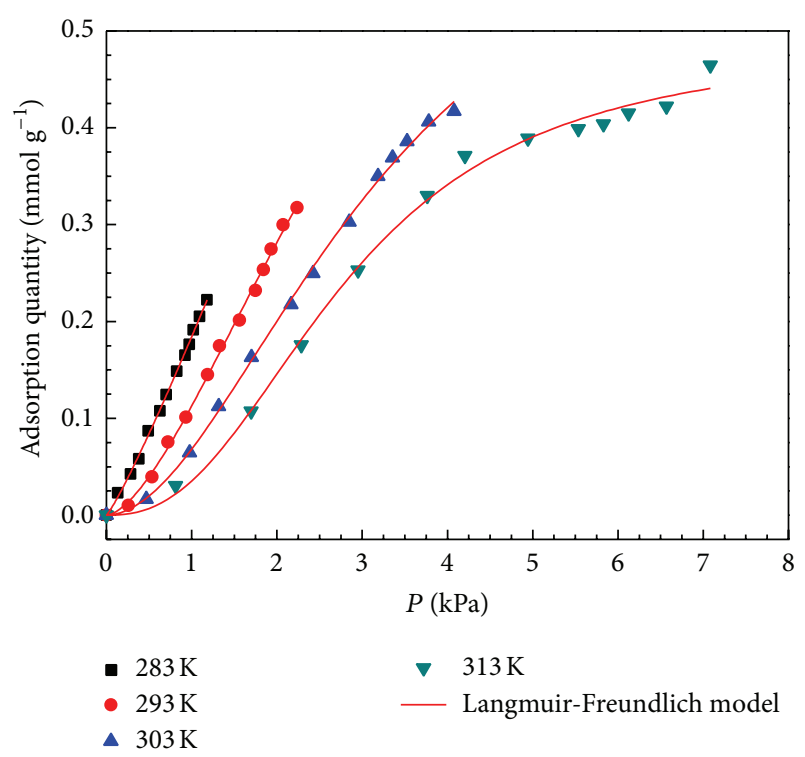

FIGURE 6: Langmuir-Freundlich isotherms of water vapor on the synthesized MFS-02 at different temperatures.

carbon-fluorine bond [28]. These two peaks are all attributed to the residual of the SURFLON S-386 after calcination. The SURFLON S-386 was composed of $-\mathrm{CF}_{2}$ groups (Figure 5). The carbon-fluorine bond is the strongest single bond in organic chemistry due to its partial ionic character [29]. It was hard to breaks the bond during the calcinations step of the synthesis process. Therefore, a portion of the SURFLON S-386 fragments might remain in the MFSs. The presence of carbon-fluorine groups on the surface of the MFSs may led to more hydrophobicity $[24,30]$.

3.2. Adsorption of Water Vapor on the MFS-02. Figure 6 shows the adsorption isotherms of water vapor on the MFS02 at $283-313 \mathrm{~K}$ measured experimentally by gravimetric method and fitted by Langmuir-Freundlich (L-F) model (2):

$$
Q_{e}=Q_{m} \frac{b_{\mathrm{L}-\mathrm{F}} P^{1 / n_{\mathrm{L}-\mathrm{F}}}}{1+b_{\mathrm{L}-\mathrm{F}} P^{1 / n_{\mathrm{LF}}}},
$$

where $Q_{e}\left(\mathrm{mmol} \mathrm{g}^{-1}\right)$ is the adsorbed amount at equilibrium pressure $P(\mathrm{mbar}), Q_{m}\left(\mathrm{mmol} \mathrm{g}^{-1}\right)$ is the saturated adsorbed amount, $b_{\mathrm{L}-\mathrm{F}}$ is the affinity coefficients $\left(\mathrm{mbar}^{-1 / n}\right)$, and $1 / n_{\mathrm{L}-\mathrm{F}}$ represents the deviations from an ideal homogeneous surface (heterogeneity factor) [31]. All the adsorption isotherms of water vapor on the MFS-02 show a typical type-V profile with an initial unfavorable adsorption isotherms at the at
TABLE 2: Langmuir-Freundlich equation fitting of water vapor adsorption on the MFS-02 at 283-313 K.

\begin{tabular}{lcccc}
\hline \multirow{2}{*}{ Temperature $(\mathrm{K})$} & \multicolumn{4}{c}{$Q_{e}=Q_{m} \frac{b_{\mathrm{L}-\mathrm{F}} P^{1 / n_{\mathrm{L}-\mathrm{F}}}}{1+b_{\mathrm{LF}} P^{1 / n_{\mathrm{L}-\mathrm{F}}}}$} \\
& $Q_{m}\left(\mathrm{mmol} \mathrm{g}^{-1}\right)$ & $b_{\mathrm{L}-\mathrm{F}}\left(\times 10^{-6}\right)$ & $1 / n_{\mathrm{L}-\mathrm{F}}$ & $R^{2}$ \\
\hline 283 & 0.43 & 2.54 & 3.65 & 0.97 \\
293 & 0.39 & 9.11 & 3.18 & 0.99 \\
303 & 0.39 & 9.18 & 3.13 & 0.99 \\
313 & 0.38 & 3.98 & 3.07 & 0.99 \\
\hline
\end{tabular}

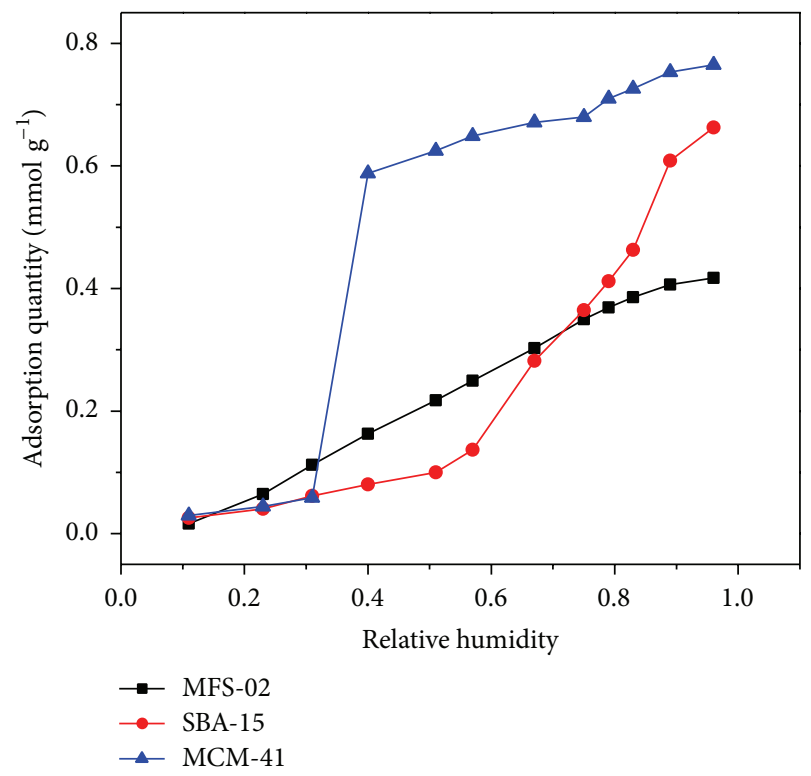

Figure 7: Comparison of the adsorption isotherms of water vapor on the MFS-02, SBA-15, and MCM-41 at $303 \mathrm{~K}$.

low absolute pressure and pore condensationat low absolute pressure. This type of isotherm of water vapour indicated a very weak adsorption interaction of water with the pores in the MFS-02. Thus, it can be seen that the surface of the MFS-02 became more hydrophobic through grafting fluorine element on the surface of the mesoporous MFS02. Meanwhile, it can also been seen that the adsorption capacity of water vapour on the MFS-02 was decreased with the increase of temperature, indicating a physisorption-type interaction. Table 2 lists the fitted parameters, $Q_{m}, b_{\mathrm{L}-\mathrm{F}}$, of the L-F model as well as the regression coefficients for linear regression of data of water vapor adsorption on the MFS02 . As shown, the experimental adsorption isotherms were well fitted by the L-F isotherm model with high regression coefficient $\left(R^{2}>0.97\right)$. Table 2 shows a slight decrease in the value of $Q_{m}$ as well as $1 / n_{\mathrm{L}-\mathrm{F}}$ as the temperature increases, suggesting a decrease in the adsorption interaction and less heterogeneous surface of the MFS-02 as the temperature increases [32].

Figure 7 shows the comparison of the adsorption isotherms of water vapor on the MFS-02, SBA-15, and MCM41 at $303 \mathrm{~K}$. As shown, the amounts of adsorbed water of these three materials were similar when the relative humidity was lower than $30 \%$, while the amount of adsorbed water on 


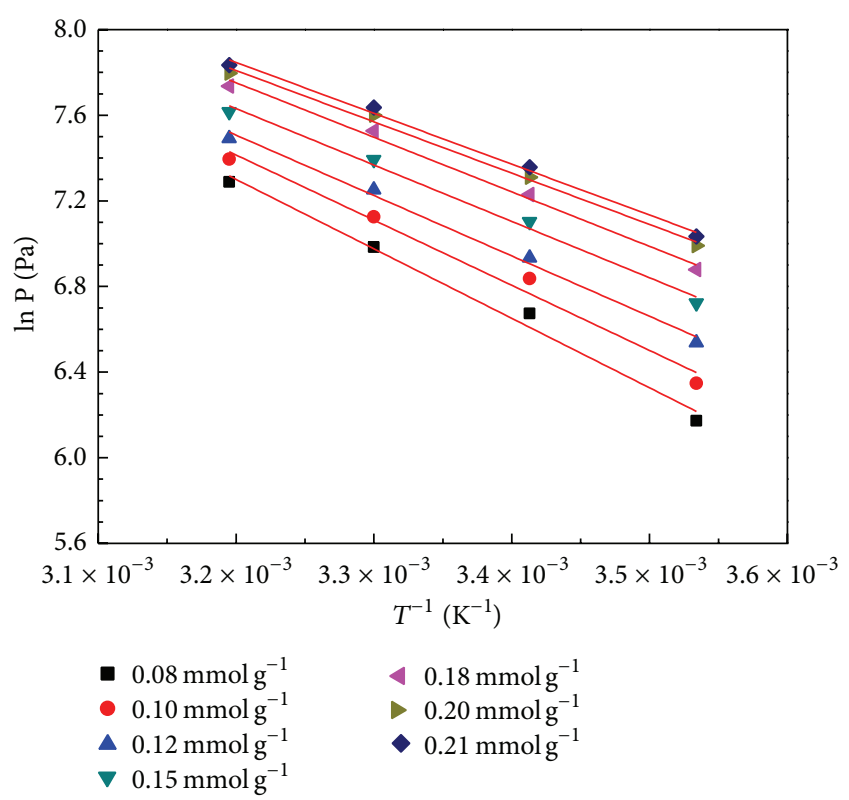

Figure 8: Temperature dependence of $\ln P$ for water vapor adsorption on the MFS- 02 .

the MFS-02 was much lower than those on the MCM-41 and SBA-15 when the relative humidity was higher than $80 \%$. It indicated that the presence of fluorine-containing group in MFS-02 might effectively increase the hydrophobic property of the porous surface, as mentioned in Figure 4.

3.3. The Isosteric Heat of Water Vapor Adsorption on the MFS. According to the method described in Section 2.4, Figure 8 can be obtained, which exhibits a series of plots of $\ln P$ versus $1 / T$ at different amounts of water vapor on the MFS-02. From the slope of the plotted straight lines, the isosteric adsorption heats $\left(\Delta H_{s}\right)$ of water vapor adsorbed on the MFS-02 under various water vapor loadings were available. Figure 9 shows the dependence of the isosteric adsorption heats on the amounts of adsorbed water vapor on the MFS-02. It indicates that the isosteric adsorption heats are in the range of $27.0-19.8 \mathrm{~kJ} \mathrm{~mol}^{-1}$, gradually decreasing with the amount of adsorbed water vapor increasing from 0.08 to $0.22 \mathrm{mmolg}^{-1}$. This means that an energetically heterogeneous surface for water vapor adsorption leads to the discrepant sorbate-sorbent interaction potential under different surface loadings [33]. The isosteric heat of water adsorption on the MFS-02 was much lower than that of water adsorption on the MCM-41 $\left(57 \mathrm{~kJ} \mathrm{~mol}^{-1}\right)$ and SBA-15 $\left(45 \mathrm{~kJ} \mathrm{~mol}^{-1}\right)$ at $Q_{m}=0.08 \mathrm{mmol} \mathrm{g}^{-1}$, indicating the more hydrophobic surface property of the MFS-02 compared to MCM-41 and SBA-15. Therefore, the MFS-02 can be used as the adsorbent for large VOCs capture or as the catalyst carrier under the condition of higher relative humidity.

\section{Conclusions}

Fluorine-containing hydrophobic mesoporous material (MFS) with high surface area was successfully synthesized

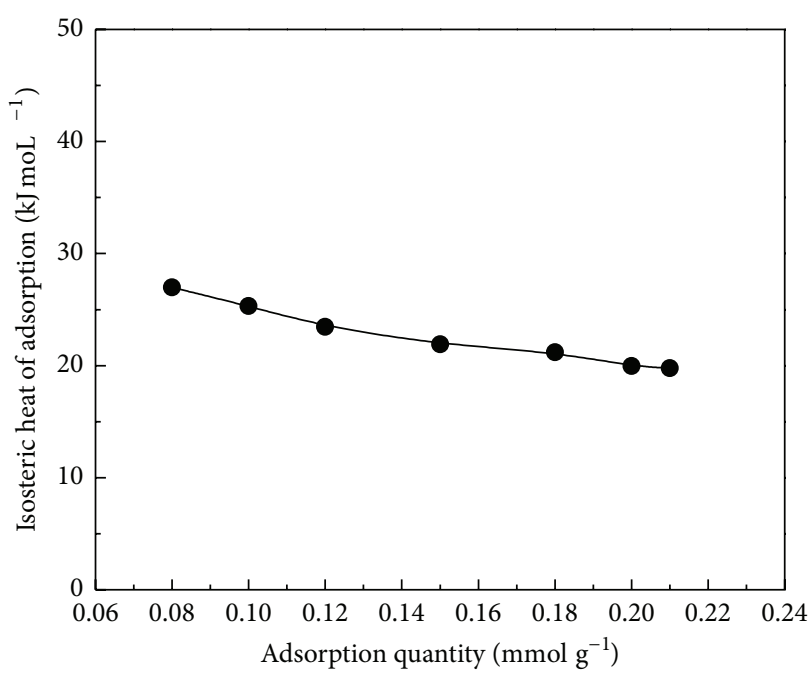

FIGURE 9: Isosteric heat of the amounts of adsorbed water vapor on the MFS-02.

with hydrothermal synthesis method by using a perfluorinated-surfactant SURFLON S-386 template. The adsorbed amount of water on the MFS-02 was much lower than that on the MCM-41 and SBA-15 at high relative humidity. The adsorption isotherms and the isosteric heat of water adsorption on the MFS-02 suggested the weak interaction between the water vapor and the MFS surface. Material characterization results showed that MFS has stronger hydrophobicity than SBA-15 and MCM-41 due to the presence of fluorine-containing group. It may be a kind of good candidate for MFS to be a good material for adsorption of large molecule and catalyst carrier under high moisture environments.

\section{Conflict of Interests}

The authors declare that there is no conflict of interests regarding the publishing of this paper.

\section{Acknowledgments}

The authors gratefully acknowledge the project supported by the National High Technology Research and Development Program of China (Grant no. 2013AA065005), the National Natural Science Foundation of China (no. 21376090), and the Project Sponsored by the Scientific Research Foundation of GuangXi University (Grant no. XGZ130963).

\section{References}

[1] A. Saral, S. Demira, and S. Yildiz, "Assessment of odorous VOCs released from a main MSW landfill site in Istanbul-Turkey via a modelling approach," Journal of Hazardous Materials, vol. 168, no. 1, pp. 338-345, 2009.

[2] N. Abbas, M. Hussaina, N. Russo, and G. Saracco, "Studies on the activity and deactivation of novel optimized $\mathrm{TiO}_{2}$ nanoparticles for the abatement of VOCs," Chemical Engineering Journal, vol. 175, pp. 330-340, 2011. 
[3] Y. S. Chen, Y. C. Hsu, C. C. Lin, C. Y. D. Tai, and H. S. Liu, "Volatile organic compounds absorption in a cross-flow rotating packed bed," Environmental Science \& Technology, vol. 42, no. 7, pp. 2631-2636, 2008.

[4] J. C. Fang, X. Chen, Q. B. Xia, H. X. Xi, and Z. Li, "Effect of relative humidity on catalytic combustion of toluene over copper based catalysts with different supports," Chinese Journal of Chemical Engineering, vol. 17, no. 5, pp. 767-772, 2009.

[5] H. Zaitan, D. Bianchi, O. Achak, and T. Chafik, "A comparative study of the adsorption and desorption of o-xylene onto bentonite clay and alumina," Journal of Hazardous Materials, vol. 153, no. 1-2, pp. 852-859, 2008.

[6] S. Modelski, A. Kołtuniewicz, and A. Witek-Krowiak, "Kinetics of VOC absorption using capillary membrane contactor," Chemical Engineering Journal, vol. 168, no. 3, pp. 1016-1023, 2011.

[7] D. E. De Vos, M. Dams, B. F. Sels, and P. A. Jacobs, "Ordered mesoporous and microporous molecular sieves functionalized with transition metal complexes as catalysts for selective organic transformations," Chemical Reviews, vol. 102, no. 10, pp. 3615$3640,2002$.

[8] M. Guillemot, J. Mijoin, S. Mignard, and P. Magnoux, "Volatile organic compounds (VOCs) removal over dual functional adsorbent/catalyst system," Applied Catalysis B: Environmental, vol. 75, no. 3-4, pp. 249-255, 2007.

[9] G. L. Athens, Y. Ein-Eli, and B. F. Chmelka, "Acid-functionalized mesostructured aluminosilica for hydrophilic proton conduction membranes," Advanced Materials, vol. 19, no. 18, pp. 25802587, 2007.

[10] M. T. Colomer, "Nanoporous anatase thin films as fast protonconducting materials," Advanced Materials, vol. 18, no. 3, pp. 371-374, 2006.

[11] G. A. Eimer, S. G. Casuscelli, C. M. Chanquia, V. Elías, M. E. Crivello, and E. R. Herrero, "The influence of Ti-loading on the acid behavior and on the catalytic efficiency of mesoporous TiMCM-41 molecular sieves," Catalysis Today, vol. 133-135, pp. 639-646, 2008.

[12] P. J. E. Harlick and A. Sayari, "Applications of pore-expanded mesoporous silica. 5. Triamine grafted material with exceptional $\mathrm{CO}_{2}$ dynamic and equilibrium adsorption performance," Industrial \& Engineering Chemistry Research, vol. 46, no. 2, pp. 446-458, 2007.

[13] W.-J. Son, J.-S. Choi, and W.-S. Ahn, "Adsorptive removal of carbon dioxide using polyethyleneimine-loaded mesoporous silica materials," Microporous and Mesoporous Materials, vol. 113, no. 1-3, pp. 31-40, 2008.

[14] R. Sanz, G. Calleja, A. Arencibia, and E. S. Sanz-Pérez, " $\mathrm{CO}_{2}$ adsorption on branched polyethyleneimine-impregnated mesoporous silica SBA-15," Applied Surface Science, vol. 256, no. 17, pp. 5323-5328, 2010.

[15] V. Zelenak, D. Halamova, L. Gaberova, E. Bloch, and P. Llewellyn, "Amine-modified SBA-12 mesoporous silica for carbon dioxide capture: effect of amine basicity on sorption properties," Microporous and Mesoporous Materials, vol. 116, no. 1-3, pp. 358-364, 2008.

[16] A. Kumar and D. Srinivas, "Selective oxidation of cyclic olefins over framework Ti-substituted, three-dimensional, mesoporous Ti-SBA-12 and Ti-SBA-16 molecular sieves," Catalysis Today, vol. 198, no. 1, pp. 59-68, 2012.

[17] D. Z. Zhao, C. Shi, X. S. Li, A. M. Zhu, and B. W.-L. Jang, "Enhanced effect of water vapor on complete oxidation of formaldehyde in air with ozone over $\mathrm{MnO}_{x}$ catalysts at room temperature," Journal of Hazardous Materials, vol. 239-240, pp. 362-369, 2012.

[18] X. Li, L. J. Wang, Q. B. Xia, Z. M. Li, and Z. Li, "Catalytic oxidation of toluene over copper and manganese based catalysts: effect of water vapor," Catalysis Communications, vol. 14, no. 1, pp. 15-19, 2011.

[19] G. Jacobs, T. K. Das, P. M. Patterson, J. Li, L. Sanchez, and B. H. Davis, "Fischer-Tropsch synthesis XAFS: XAFS studies of the effect of water on a Pt-promoted $\mathrm{Co} / \mathrm{Al}_{2} \mathrm{O}_{3}$ catalyst," Applied Catalysis A: General, vol. 247, no. 2, pp. 335-343, 2003.

[20] Q.-H. Xia, K. Hidajat, and S. Kawi, "Improvement of the hydrothermal stability of fluorinated MCM-41 material," Materials Letters, vol. 42, no. 1, pp. 102-107, 2000.

[21] D. Shen, M. Bülow, F. Siperstein, M. Engelhard, and A. L. Myers, "Comparison of experimental techniques for measuring isosteric heat of adsorption," Adsorption, vol. 6, no. 4, pp. 275286, 2000.

[22] A. Ansón, M. A. Callejas, A. M. Benito et al., "Hydrogen adsorption studies on single wall carbon nanotubes," Carbon, vol. 42, no. 7, pp. 1237-1241, 2004.

[23] Z. X. Zhao, X. M. Li, and Z. Li, "Adsorption equilibrium and kinetics of $\mathrm{p}$-xylene on chromium-based metal organic framework MIL-101," Chemical Engineering Journal, vol. 173, no. 1, pp. 150-157, 2011.

[24] S. C. Sharma, H. Kunieda, J. Esquena, and C. Rodríguez Abreu, "Phase behavior and preparation of mesoporous silica in aqueous mixtures of fluorinated surfactant and hydrophobic fluorinated polymer," Journal of Colloid and Interface Science, vol. 299, no. 1, pp. 297-304, 2006.

[25] Y.-L. Su, J. Wang, and H.-Z. Liu, "FTIR spectroscopic study on effects of temperature and polymer composition on the structural properties of PEO-PPO-PEO block copolymer micelles," Langmuir, vol. 18, no. 14, pp. 5370-5374, 2002.

[26] Z. Sarbak, "FT-IR studies on coke formation over low loaded Mo catalyst supported on $\mathrm{Al}_{2} \mathrm{O}_{3}$," Reaction Kinetics and Catalysis Letters, vol. 84, no. 2, pp. 263-270, 2005.

[27] J. W. Yi, Y. H. Lee, and B. Farouk, "Low dielectric fluorinated amorphous carbon thin films grown from $\mathrm{C}_{6} \mathrm{~F}_{6}$ and Ar plasma," Thin Solid Films, vol. 374, no. 1, pp. 103-108, 2000.

[28] J. F. Kang, A. Ulman, R. Jordan, and D. G. Kurth, "Optically induced band shifts in infrared spectra of mixed self-assembled monolayers of biphenyl thiols," Langmuir, vol. 15, no. 17, pp. $5555-5559,1999$.

[29] D. O'Hagan, “Understanding organofluorine chemistry. An introduction to the C-F bond," Chemical Society Reviews, vol. 37, pp. 308-319, 2008.

[30] J. L. Blin and M. J. Stébé, "Effect of fluorocarbon addition on the structure and pore diameter of mesoporous materials prepared with a fluorinated surfactant," Microporous and Mesoporous Materials, vol. 87, no. 1, pp. 67-76, 2005.

[31] A. Anson, Y. Wang, C. C. H. Lin, T. M. Kuznicki, and S. M. Kuznicki, "Adsorption of ethane and ethylene on modified ETS10," Chemical Engineering Science, vol. 63, no. 16, pp. 4171-4175, 2008.

[32] A. R. Zimmerman, K. W. Goyne, J. Chorover, S. Komarneni, and S. L. Brantley, "Mineral mesopore effects on nitrogenous organic matter adsorption," Organic Geochemistry, vol. 35, no. 3, pp. 355-375, 2004.

[33] R. T. Yang, Adsorbents: Fundamentals and Applications, WileyInterscience, 1st edition, 2003. 

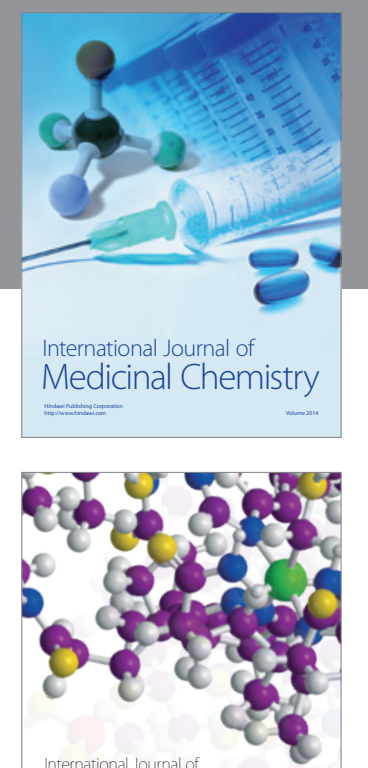

\section{Carbohydrate} Chemistry

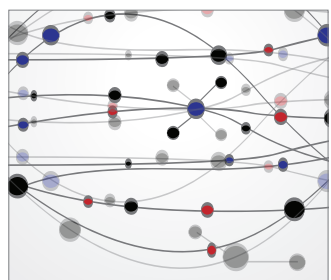

The Scientific World Journal
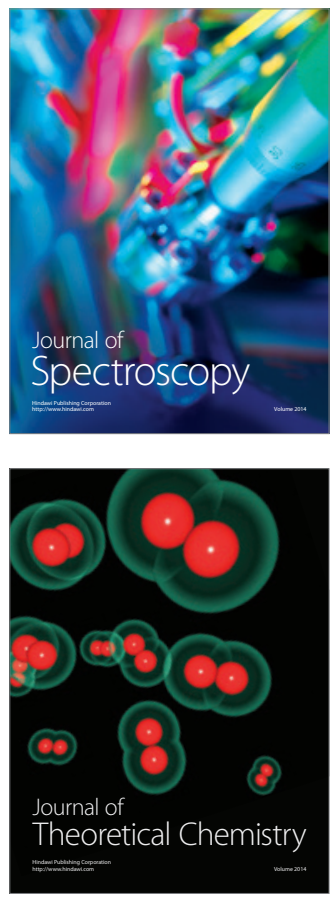
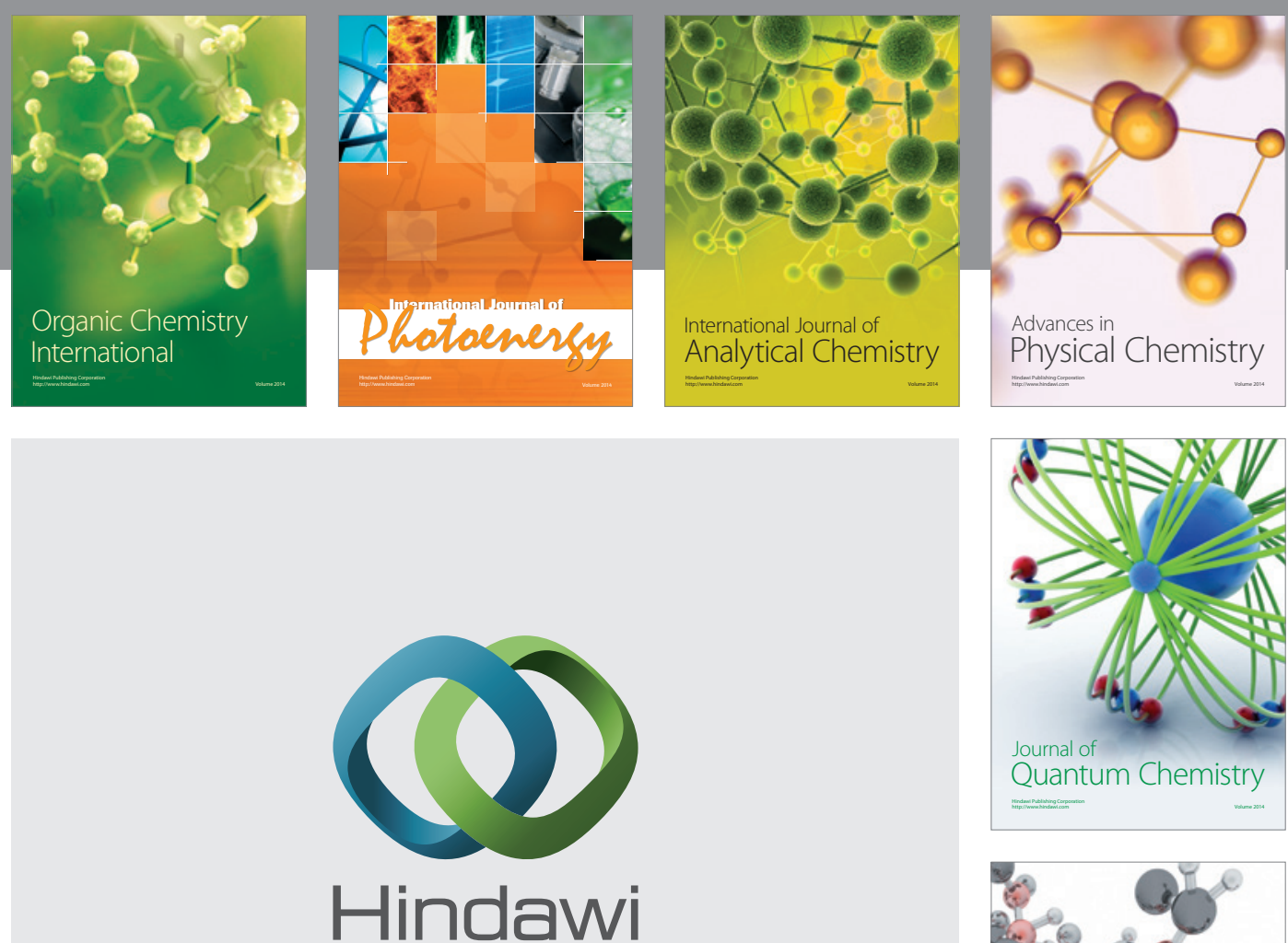

Submit your manuscripts at

http://www.hindawi.com

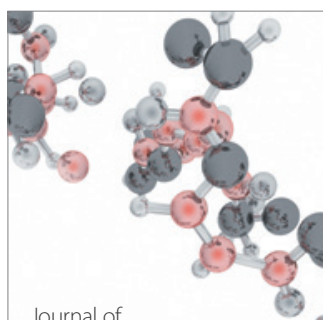

Analytical Methods

in Chemistry

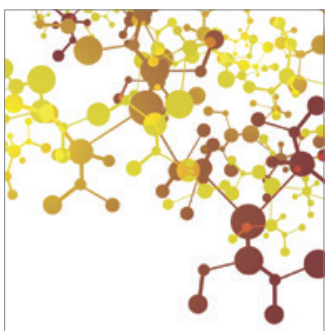

Journal of

Applied Chemistry

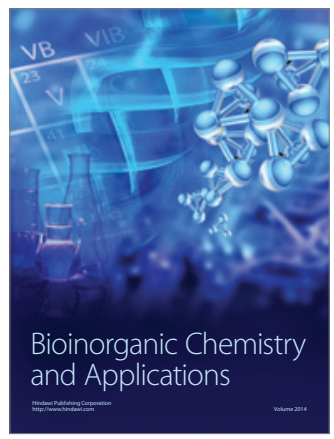

Inorganic Chemistry
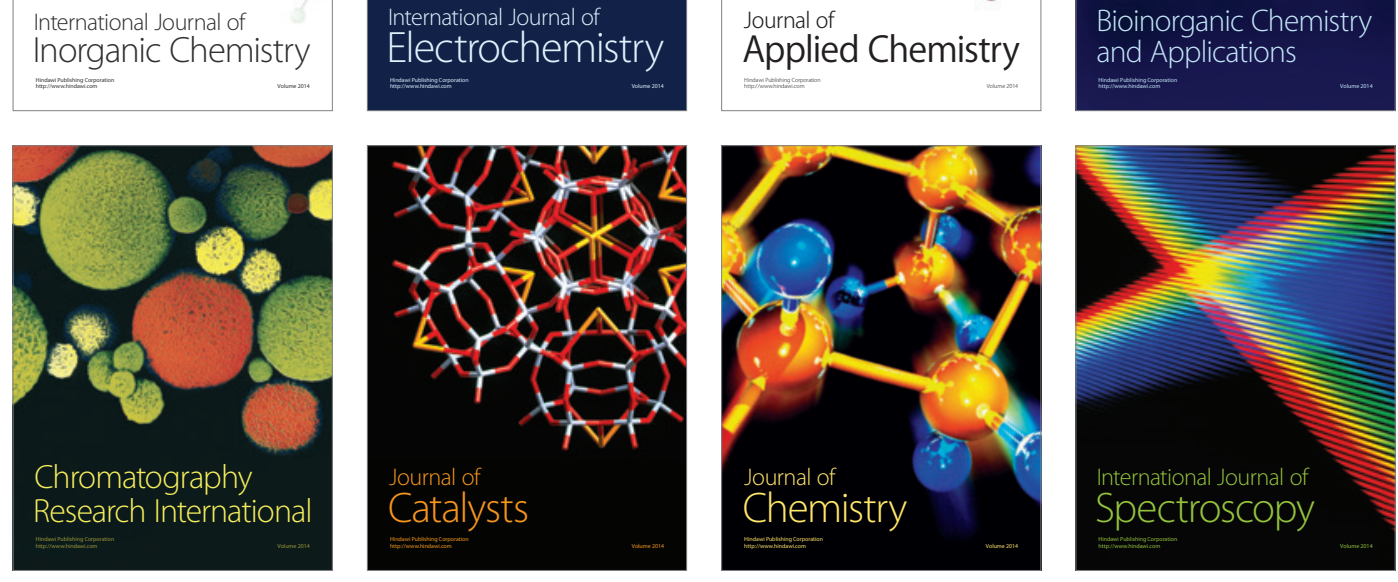